\title{
Tunable wideband bandstop acoustic filter based on two-dimensional multiphysical phenomena periodic systems
}

\author{
V. Romero-García, ${ }^{1,2, a)}$ J. V. Sánchez-Pérez, ${ }^{2}$ and L. M. Garcia-Raffi ${ }^{3}$ \\ ${ }^{1}$ Instituto para la Gestión Integrada de zonas Costeras, Universidad Politécnica de Valencia, 46730 \\ Gandia, Spain \\ ${ }^{2}$ Centro de Tecnologías Físicas: Acústica, Materiales y Astrofísica, Universidad Politécnica de Valencia, \\ Camino de Vera s/n, 46022 Valencia, Spain \\ ${ }^{3}$ Instituto Universitario de Matemática Pura y Aplicada, Universidad Politécnica de Valencia, Camino de \\ Vera s/n, 46022 Valencia, Spain
}

(Received 12 February 2011; accepted 10 May 2011; published online 6 July 2011)

\begin{abstract}
The physical properties of a periodic distribution of absorbent resonators is used in this work to design a tunable wideband bandstop acoustic filter. Analytical and numerical simulations as well as experimental validations show that the control of the resonances and the absorption of the scatterers along with their periodic arrangement in air introduce high technological possibilities to control noise. Sound manipulation is perhaps the most obvious application of the structures presented in this work. We apply this methodology to develop a device as an alternative to the conventional acoustic barriers with several properties from the acoustical point of view but also with additional esthetic and constructive characteristics. (C) 2011 American Institute of Physics. [doi:10.1063/1.3599886]
\end{abstract}

\section{INTRODUCTION}

The interesting propagation properties of electromagnetic waves inside an inhomogeneous medium with periodic modulation of its dielectric properties, were simultaneously emphasized by Yablonovitch ${ }^{1}$ and John. ${ }^{2}$ These periodic systems exhibit ranges of frequencies, related to the periodicity of the structure, where there is no wave propagation. In analogy with the electronic bandgap in semiconductor crystals, these ranges of frequencies were called bandgaps (BGs). Motivated by these results, an increasing interest in the comparable process of acoustic wave propagation in inhomogeneous periodic acoustic medium appeared. Several theoretical works started the analysis of periodic arrays made of isotropic solids embedded in an elastic background which was also isotropic. ${ }^{3,4} \mathrm{By}$ analogy with the electromagnetic case, these periodic arrangements present BGs for elastic waves and they were called phononic crystals (PCs). It is said that if one of the elastic materials in the PCs is a fluid medium, then PCs are called sonic crystals (SCs). Several studies discuss the similarities and differences between them. ${ }^{5,6}$ Since the acoustical properties of SCs were measured in a minimalist sculpture, ${ }^{7}$ much research interest, both experimental and theoretical, has been focused on the existence of complete elastic/acoustic BGs, opening possibilities to interesting applications such as elastic/acoustic filters, noise control, improvements in the design of transducers, as well as for the study of pure physics phenomena.

The possibility to manipulate the sound by means of SCs motivated the idea of using these periodic acoustic media as attenuation devices as, for example, an alternative to noise barriers. ${ }^{8}$ However, from the acoustical point of view, the mere existence of the BGs is not sufficient to use

\footnotetext{
${ }^{\text {a) }}$ Author to whom correspondence should be addressed. Electronic mail: virogar1@mat.upv.es
}

SCs as noise barriers because both the size and position of these BGs depend on several factors such as the angle of incidence of the wave or the arrangement of the scatterers. To avoid these problems, some strategies to increase the acoustical properties of SCs have been developed in the last few years. First, some authors have studied new and more efficient arrangements of scatterers out of the classical crystalline ones, such as quasicrystals, ${ }^{9}$ quasiordered structures ${ }^{10,11}$ or quasifractal arrangements. ${ }^{12}$ Another strategy is the use of scatterers with additional properties. This last one can reduce the angular dependence of the attenuation achieved by the periodic arrangement and increases both the level and the range of the attenuated frequencies. In this sense, some authors have designed rigid scatterers combined with absorbent materials in which both the multiple scattering and the absorption effects appear in the periodic arrangement. ${ }^{13}$ Moreover the study of SCs built with scatterers with resonant effects has been done, obtaining good results especially at frequencies below the BGs. ${ }^{14,15}$ Several works have been very recently developed mixing these effects in order to increase the properties of the SCs as noise barriers. ${ }^{16,17}$

In this work we present a wideband bandstop acoustic filter for the range of the audible frequencies based on the combination of scattering, resonances and absorption in a $2 \mathrm{D}$ periodic array of scatterers in air. The building blocks of these systems are scatterers made of a combination of rigid, absorbent, and resonant cavities. Figure 1(a) shows the transversal section of the scatterer considered in this work. A cylindrical rigid wall is covered by a porous material. A slit is made all along the cylinder in such a way the shape of the scatterer presents a resonant cavity, thus these scatterers are based on the concept of split ring resonator (SRR) introduced by Pendry et al. in 1999 (Ref. 18) in the context of the electromagnetism.

The square periodicity of the 2D system is shown in Fig. 1(b). This SC is characterized by two parameters: the lattice 


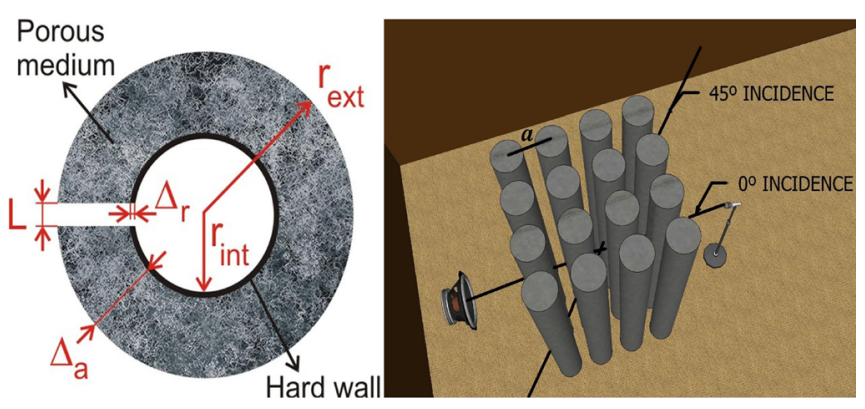

FIG. 1. (Color online) (a),(b) Schematic view of both the scatterer and the SC configurations of the device presented. (a) The transversal view of the 1 $\mathrm{m}$ long scatterer considered. The inner radius is $r_{\text {int }}=0.095 \mathrm{~m}$, the exterior radius is $r_{\text {ext }}=0.14 \mathrm{~m}$, the aperture $L=0.02 \mathrm{~m}$, the rigid wall thickness $\Delta_{r}=0.005 \mathrm{~m}$ and the absorbent covering thickness $\Delta_{a}=0.04 \mathrm{~m}$. (b) The size of the designed device is $4 a \times 4 a$. The scatterers are arranged in a square array with lattice constant $a=0.33 \mathrm{~m}$. For this square periodicity the main directions of symmetry of the SC are $\Gamma X\left(0^{\circ}\right)$ and $\Gamma M\left(45^{\circ}\right)$.

constant $a$, separation between scatterers, and the filling fraction $f f$, the volume occupied by the scatterers in the SC. For this periodicity, the main directions of symmetry are $\Gamma X\left(0^{\circ}\right)$ and $\Gamma M$ $\left(45^{\circ}\right)$. In this system, the periodic distribution of the rigid properties of the scatterers leads to the phenomenon of the BG, the absorbent material of each scatterer gives a threshold of attenuation in the audible frequencies, and finally the resonant cavities introduce attenuation in relation with the resonances. But all these effects can be fitted by introducing high technology procedures in the design of devices to control the noise. We have progressively studied both theoretically and experimentally the acoustic effect of each component of the scatterer on the frequency response of the whole system. From the theoretical point of view we analyze the effect of each phenomenon by means of several theoretical and numerical techniques.

Thus, the infinite periodic distribution of an array of rigid scatterers is analyzed by means of the dispersion relation using the plane wave expansion (PWE) method. This method uses the periodicity of the system and the Bloch theorem to solve the wave equation, obtaining a simple eigenvalue problem relating to the wave vector and the frequency of the incident wave. PWE allows us to understand the transmission properties of the wave inside the crystalline systems. However, in the real situations the finite sample effects have to be taken into account. In this sense, the multiple scattering theory (MST) is introduced as a methodology to study the scattering in a finite distribution of cylindrical scatterers. The methodology is developed and used for two cases, rigid and absorbent scatterers, in order to compare and separate the contributions of both the absorbent material and the effect of the periodicity of the array in the total attenuation obtained. The results are compared with those obtained using PWE. The case of the resonant effect, due to the complexity of the shape of the scatterers because of the existence of the split all along the cylinders, has been analyzed numerically using the finite elements method (FEM). The combined effect of scattering, resonances and absorption in the definitive SC device is studied both numerically and experimentally. Finally, the main applications of such a structures and the main conclusions of the work are summarized in the last sections.

\section{THEORETICAL METHODS}

A wide range of mathematical techniques are now available for the resolution of problems involving the interaction of waves with arrays of scatterers. This section draws together the methods used in this work: PWE, MST, and FEM.

In order to show the main results obtained by each theoretical method explained in this section, we have applied them to study the propagation properties of a SC made of rigid or absorbent cylindrical scatterers with $r=0.1 \mathrm{~m}$, similar to that shown in Fig. 1(a) but with $L=0$. These scatterers are arranged in a square array with $a=0.33 \mathrm{~m}$. The filling fraction of the array is $f f=\pi r^{2} / a^{2}=0.2885$ (28.85\%). This preliminary study allow us to understand the acoustic properties of the definitive designed device.

Propagation of sound is described by the equation

$$
\frac{1}{\rho c^{2}} \frac{\partial^{2} p}{\partial t^{2}}=\nabla\left(\frac{1}{\rho} \nabla p\right),
$$

where $c$ is the sound velocity, $\rho$ is the density of the medium, and $p$ is the pressure. In this work we consider harmonic temporal dependence to solve for both scattering and eigenvalue problems.

\section{A. Eigenvalue problem: Dispersion relation}

The dispersion relation of a 2D SC made of rigid cylindrical scatterers can be obtained by means of the solution of the eigenvalue problem using the PWE. These bands structures are a good representation to understand the dispersion of waves inside such crystalline systems. This method is valid only for infinite periodic crystals.

In analogy to electron waves in a crystal, waves transmission inside periodic systems should be described using the bands theory. This idea was first introduced in 1987 (Refs. 1, 2) and then the concepts of Bloch waves, dispersion relations, Brillouin zones and so on, can be applied to the case of all kind of periodic systems: photonic, phononic and sonic crystals. ${ }^{6,19-21}$ Very recent works have shown extensions of the PWE to solve the inverse complex value problem $k(\omega)$ (Refs. 22, 23, 27) as well as to introduce effects of damping in the calculation of the dispersion relation. ${ }^{24-26}$

\section{Plane wave expansion (PWE)}

In this section, an array of straight, infinite cylinders made of an isotropic solid $A$, embedded in an acoustic isotropic background $B$ has been considered. There is translational invariance in the direction $z$ parallel to the cylinders and the system has 2D periodicity in the transverse plane. Thus, this assumption implies an infinite medium in order to obtain its propagation properties. By using this periodicity, it is possible to expand the properties of the medium in Fourier series,

$$
\begin{aligned}
& \sigma=\frac{1}{\rho(\vec{r})}=\sum_{\vec{G}} \sigma_{\vec{k}}(\vec{G}) e^{l \vec{G} \vec{r}}, \\
& \eta=\frac{1}{\beta(\vec{r})}=\sum_{\vec{G}} \eta_{\vec{k}}(\vec{G}) e^{l \vec{G} \vec{r}},
\end{aligned}
$$


TABLE I. Directions of incidence, ranges of $\vec{k}$, and ranges of phase changes $\vec{k} \cdot \vec{R}_{1}$ and $\vec{k} \cdot \vec{R}_{2}$, for each of the segments required to traverse the boundary of the irreducible first Brillouin zone for square lattice.

\begin{tabular}{llll}
\hline \hline & \multicolumn{3}{c}{ Square lattice } \\
\cline { 2 - 4 } Direction & $\vec{k}$ & $\vec{k} \cdot \vec{R}_{1}$ & $\vec{k} \cdot \vec{R}_{2}$ \\
\hline$\Gamma X$ & {$[(0,0)(0, \pi / a)]$} & {$[0, \pi / a]$} & {$[0,0]$} \\
$\mathrm{XM}$ & {$[(\pi / a, 0)(\pi / a, \pi / a)]$} & {$[\pi / a, \pi / a]$} & {$[0, \pi / a]$} \\
$M \Gamma$ & {$[(\pi / a, \pi / a)(0,0)]$} & {$[\pi / a, 0]$} & {$[\pi / a, 0]$} \\
\hline \hline
\end{tabular}

where $\vec{G}$ is the $2 \mathrm{D}$ reciprocal-lattice vector, $\vec{G}=\left(G_{1}, G_{2}\right.$, $\left.G_{3}\right)=\left(2 \pi m / a_{1}, 2 \pi n / a_{2}, 0\right)$ for square periodicity, and $\beta(\vec{r})=\rho(\vec{r}) c(\vec{r})^{2}$ is the bulk modulus. The pressure $p$ can be obtained by applying the Bloch theorem and harmonic temporal dependence,

$$
p(\vec{r}, t)=e^{l(\vec{k} \vec{r}-\omega t)} \sum_{\vec{G}} p_{k}(\vec{G}) e^{l \vec{G} \vec{r}}
$$

Using Eqs. (2), (3), (4), and (1) we obtain ${ }^{21}$

$$
\sum_{\vec{G}^{\prime}}\left[(\vec{k}+\vec{G}) \sigma_{k}\left(\vec{G}-\vec{G}^{\prime}\right)\left(\vec{k}+\vec{G}^{\prime}\right)-\omega^{2} \eta_{\vec{k}}\left(\vec{G}-\vec{G}^{\prime}\right)\right] p_{\vec{k}}\left(\vec{G}^{\prime}\right)=0 .
$$

For $\vec{G}$ taking all the possible values, Eq. (5) constitutes a set of linear, homogeneous equations for the eigenvectors $p_{\vec{k}(\vec{G})}$ and the eigenfrequencies $\omega(\vec{k})$.

By solving the system given in Eq. (5) for each Bloch vector in the irreducible area of the first Brillouin, zone the eigenvalues $\omega^{2}$ are obtained, and they can be used to represent the band structures or dispersion relation $\omega(\vec{k})$ in the periodic system. Table I shows the directions of incidence, ranges of $\vec{k}$, and ranges of phase changes, $\vec{k} \cdot \vec{R}_{1}$ and $\vec{k} \cdot \overrightarrow{R_{2}}$, for each of the segments required to traverse the boundary of the irreducible first Brillouin zone for the square lattice.
In Fig. 2(a) one can observe the bands structure of the selected SC. For the calculations we have used 841 plane waves showing a good convergence of the PWE. The frequencies are represented versus the Bloch vector in the first Brillouin zone which is in relation with the incident direction of the wave. Each black line represents a propagating band, i.e., allowed modes inside the periodic structure. Note that for the $\Gamma X$ direction there is a range of frequencies (marked with horizontal dashed lines) in which there is no propagation. This forbidden frequency range for a specific incident direction is known as a pseudogap. In this angle of incidence and for this frequencies the SC works as an acoustic filter.

\section{Finite element methods, bounded problem, rigid scatterers}

For solving the problem using FEM, it is necessary to define the symmetry, discretize the domain and consider the boundary conditions. In the boundary of each scatterer both the continuity of the pressure and the velocity are considered. As we have explained before, for rigid scatterers we can use both the Neumann boundary condition and Bloch theorem due to the translational symmetry. The properties of the Bloch states constrain the solution to a unit cell with Bloch vectors in the first Brillouin zone. These features transform the unit cell in a bounded domain to solve the problem with the next boundary condition at the borders of the unit cell,

$$
p(\vec{r}+\vec{R})=p(\vec{r}) e^{i \vec{k} \vec{R}},
$$

where $k$ is the Bloch vector and it scans the first irreducible Brillouin zone (see Table I for square periodicity).

The red circles in Fig. 2(a) show the bands structure predicted using FEM. The unit cell has been discretized using $15 \times 10^{3}$ elements. One can compare the very good agreement between the FEM results with the ones obtained using PWE.

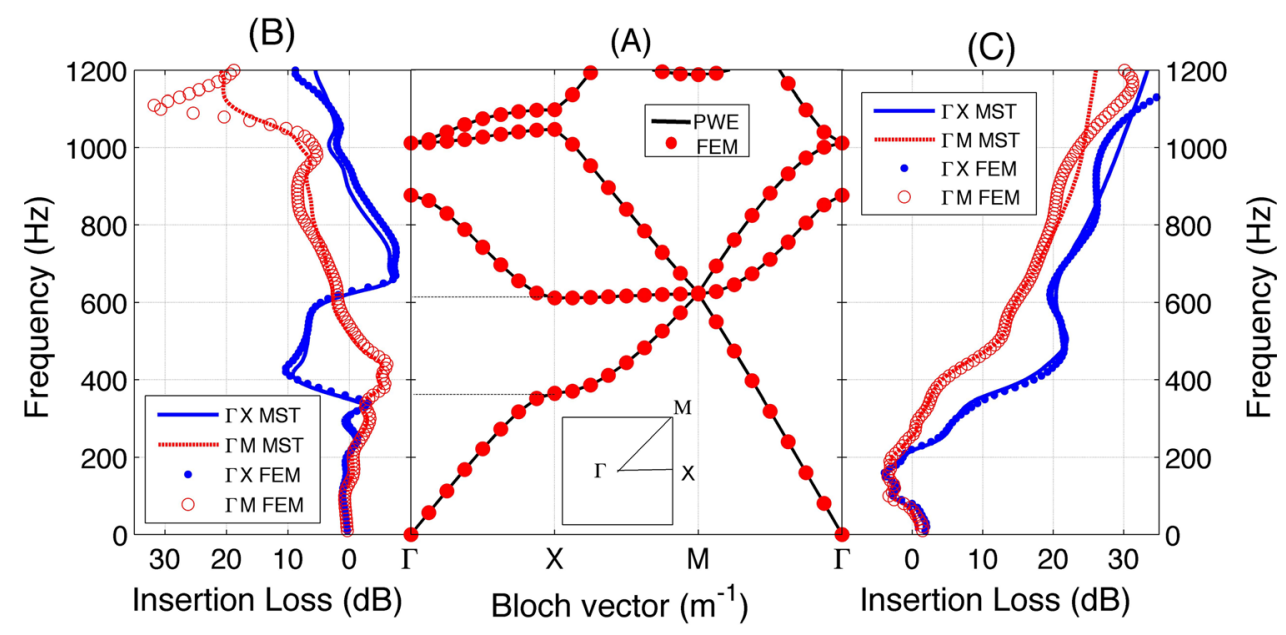

FIG. 2. (Color online) Theoretical propagation properties of a SC made of rigid or absorbent scatters obtained with the three methods used in this work. (a) Bands structure (or dispersion relation) of a SC made of rigid scatterers with $a=0.33 \mathrm{~m}$ and $r=0.1 \mathrm{~m}$. Black lines represent the analytical predictions calculated using PWE. Red dots show the numerical predictions calculated using FEM. Horizontal dashed lines represent a pseudogap. (b) and (c): corresponding scattering problem calculated for a SC of size $4 a \times 4 a$ made of rigid or absorbent scatterers, respectively. The SC is placed at $0.4 \mathrm{~m}$ from the source and centered with respect to the source-receiver line. The site of the receiver is $(2.49,0) \mathrm{m}$. Blue continuous line (blue dots) represents the attenuation spectrum for the $\Gamma X$ direction predicted by MST (FEM). Red dashed line (red open circles) represents the attenuation spectrum for the $\Gamma M$ direction predicted by MST (FEM). 


\section{B. Scattering problem}

\section{Multiple scattering theory (MST)}

In real situations the finite sample effects have to be taken into account. Multiple scattering Theory gives the possibility to study the scattering problem in these finite structures. Motivated by the work of Tournat et al. ${ }^{28}$ in which scatterers with a mesoscopic scale much larger than the microscopic scale are placed in a porous medium as host material, we briefly present here the multiple scattering of a $2 \mathrm{D}$ array of scatterers made of a rigid core covered with a layer of absorbing materials. The inner rigid core is a cylinder with radius $r_{\text {in }}$, and the covering of the absorbing material has a thickness $t$, so that the external radius is $r_{\mathrm{ext}}=r_{\text {in }}+t$. Other works ${ }^{13}$ show similar methodology as in this section.

Absorbing materials usually present a complex impedance, $Z_{c}(\omega)$, and complex propagation constant, $k_{c}(\omega)$, both being frequency dependent. Therefore we will consider two different boundary conditions: one corresponding to the rigid core and the other across the interface between the absorbing covering of the scatterer and the surrounding medium. The formalism developed in this section shows a general procedure, independently from the expressions of $Z_{c}$ and $k_{c}$ used to model the absorbing material. The porous material will be described by means of the Delany-Bazely model, then

$$
\begin{gathered}
Z_{c}(\nu)=1+0.0571\left(\frac{\rho_{0} \nu}{R}\right)^{0.754}-\imath 0.087\left(\frac{\rho_{0} \nu}{R}\right)^{-0.732}, \\
k_{c}(\nu)=k_{0}\left[1+0.0928\left(\frac{\rho_{0} \nu}{R}\right)^{-0.7}+\imath 0.189\left(\frac{\rho_{0} \nu}{R}\right)^{-0.597}\right],
\end{gathered}
$$

where $\rho_{0}, c_{0}$ represent the density and the sound velocity of the air, respectively; $k_{0}$ is the wave number of the wave propagating in air; $R$ is the flow resistivity; and $\nu$ is the frequency of sound $(\omega=2 \pi \nu)$. Here $R=23000 \mathrm{~Pa} \mathrm{~s} \mathrm{~m}^{-2}$ corresponds to the woolen felt covering. In order to avoid nonphysical results the signs of the imaginary part of $Z_{c}$ and $k_{c}$ have been properly selected. This improves the results shown in previous works. ${ }^{13}$ This model presents some ranges of applicability dependent on both the resistivity of flow and the frequency. For the range of values of these parameters, the Delany-Bazley model works properly in the range of frequencies $186<\nu<18700 \mathrm{~Hz}$, which is basically the range of frequencies in the audible range $(20-22000 \mathrm{~Hz})$.

An acoustic source transmitting white noise is placed at point $\vec{r}_{s}$, located at some distance from the system of scatterers. For the sake of simplicity, without compromising generality, we approximate the acoustic source as a line source located at origin, i.e., $\vec{r}_{s}=\overrightarrow{0}$. The acoustic wave emitted by such a source follows the equation in cylindrical coordinates:

$$
P_{0}(\vec{r})=\imath \pi H_{0}(k r),
$$

where $H_{0}$ is the zeroth order Hankel function of the first kind. The solution represents a line source located at origin.

We consider $N$ straight scatterers located at $\vec{r}_{i}=\left(r_{i}, \theta_{i}\right)$ with $i=1,2, \ldots, N$ to form either a regular lattice or a random array perpendicular to the $x-y$ plane. The scatterers are parallel to the $z$-axis, then since the boundary conditions and the geometry do not change with $z$, the problem can be reduced to two uncoupled problems for the scalar Helmholtz equation. The final wave reaches a receiver located at $\vec{r}_{r}$ and it is formed by the sum of the direct wave from the source and the scattered waves from all the scatterers.

The incident wave over $i$ th scatterer at a point $\vec{r}$ outside the scatterer is

$$
P_{i n}^{i}(\vec{r})=\sum_{n=-\infty}^{\infty} B_{n}^{i} J_{n}\left(k\left|\vec{r}-\vec{r}_{i}\right|\right) e^{i n \phi_{\vec{r}-\vec{r}_{i}}}
$$

On the other hand, the scattered wave produced by the $i$ th scatterer at a point $\vec{r}$ outside the scatterer is

$$
P_{s c}^{i}\left(\vec{r}, \vec{r}_{i}\right)=\sum_{n=-\infty}^{\infty} l \pi A_{n}^{i} H_{n}^{(1)}\left(k\left|\vec{r}-\vec{r}_{i}\right|\right) e^{i n \phi_{\vec{r}-\vec{r}_{i}}},
$$

where $H_{n}$ is the $n$th order Hankel function of the first kind, and $J_{n}$ is the $n$th order Bessel function of the first kind.

The wave transmitted within the absorbing material of the $i$ th scatterer at a point $\vec{r}$ inside the absorbing layer is

$$
\begin{aligned}
P_{\text {int }}^{i}\left(\vec{r}, \vec{r}_{i}\right)= & \sum_{n=-\infty}^{\infty} A_{n}^{i}\left\{X_{n}^{i} H_{n}^{(1)}\left(k_{c}(\omega)\left|\vec{r}-\vec{r}_{i}\right|\right)\right. \\
& +Y_{n}^{i} J_{n}\left(k_{c}(\omega)\left|\vec{r}-\vec{r}_{i}\right|\right) e^{i n \phi_{\vec{r}-\vec{r}_{i}}},
\end{aligned}
$$

where $X_{n}^{i}$ and $Y_{n}^{i}$ are two coefficients to be calculated later with appropriate boundary conditions. Then, the external wave outside the $i$ th scatterer at a point $\vec{r}$ outside the scatterer is

$P_{\text {ext }}\left(\vec{r}, \vec{r}_{i}\right)=\sum_{n=-\infty}^{\infty}\left[B_{n}^{i} J_{n}\left(k\left|\vec{r}-\vec{r}_{i}\right|\right)+\imath \pi A_{n}^{i} H_{n}^{(1)}\left(k\left|\vec{r}-\vec{r}_{i}\right|\right)\right] e^{i n \phi_{\vec{r}-\vec{r}_{i}}}$

Due to the scatterers considered in this Section, the problem presents two different kinds of boundary conditions. In the walls of the rigid core, one can consider Neumann boundary conditions. However, in the absorbing material-host medium interface, one should consider the continuity of the pressure and the velocity. Thus, the boundary condition in the rigid wall, $\Gamma_{i}$, inside $i$ th scatterer is

$$
\left.\frac{\partial P_{i n t}^{i}}{\partial n}\right|_{\Gamma_{i}}=0
$$

and the boundary conditions in the external interface, $\Omega_{i}$, of the scatterer are

$$
\begin{gathered}
\left.p_{\text {ext }}^{i}\right|_{\partial \Omega_{i}}=\left.p_{i n t}^{i}\right|_{\partial \Omega_{i},} \\
\left.\frac{Z_{c}(\omega) k_{c}(\omega)}{k_{0}} \frac{\partial p_{e x t}}{\partial n}\right|_{\partial \Omega_{i}}=\left.\frac{\partial p_{i n t}}{\partial n}\right|_{\partial \Omega_{i}},
\end{gathered}
$$

where $\partial \boldsymbol{\Omega}_{i}$ is the boundary of the $i$ th scatterer, $k_{0}$ is the wave number in the host medium, $k_{c}(\omega)$ and $Z_{c}(\omega)$ are the propagation constant and the impedance of the absorbing material of the scatterer $i$.

By applying the boundary condition [Eq. (14)] in $\Gamma_{i}$, we can obtain a simple relation between coefficients $X_{n}^{i}$ and $Y_{n}^{i}$ : 


$$
\begin{gathered}
Y_{n}^{i}=X_{n}^{i} T_{n}^{i}, \\
T_{n}^{i}=-\frac{H_{n}^{\prime}\left(k_{c}(\omega) r_{i n}^{i}\right)}{J_{n}^{\prime}\left(k_{c}(\omega) r_{i n}^{i}\right)},
\end{gathered}
$$

where the prime as superscript represents the derivative with respect to the normal of the surface

Finally, applying the boundary condition [Eq. (16)] at the $\partial \Omega_{i}$ interfaces, we get

$$
B_{n}^{i}=\imath \pi Z_{n}^{i} A_{n}^{i}
$$

where

$$
\begin{gathered}
Z_{n}^{i}=-\frac{f(\omega) H_{n}^{\prime}\left(k r_{\text {out }}^{i}\right)-H_{n}\left(k r_{\text {out }}^{i}\right)}{f(\omega) J_{n}^{\prime}\left(k r_{\text {out }}^{i}\right)-J_{n}\left(k r_{\text {out }}^{i}\right)}, \\
f(\omega)=\frac{Z_{c}(\omega) k_{c}(\omega)}{k}, \\
\frac{H_{n}\left(k_{c}(\omega) r_{\text {out }}^{i}\right)+T_{n}^{i} J_{n}\left(k_{c}(\omega) r_{\text {out }}^{i}\right)}{H_{n}^{\prime}\left(k_{c}(\omega) r_{\text {out }}^{i}\right)+T_{n}^{i} J_{n}^{\prime}\left(k_{c}(\omega) r_{\text {out }}^{i}\right)} .
\end{gathered}
$$

Note that the previous equations also reproduce the case of rigid scatterers. If the absorbing cover is not considered, then $r_{\text {out }}=r_{\text {in }}, Z_{c}(\omega)=1$, and $k_{c}(\omega)=k$ and the equations obtained are the same as the ones previously obtained by several authors. ${ }^{29}$

The attenuation spectrum of an arrangement of scatterers is obtained by the representation of the insertion loss (IL) which is the difference between the sound level recorded with and without the sample. Note that throughout this work the IL is calculated as

$$
\mathrm{IL}=20 \log _{10} \frac{\left|P_{0}\right|}{|P|},
$$

where $P$ is calculated as

$$
\begin{aligned}
P(\vec{r})= & \imath \pi H_{0}(k r) \\
& +\sum_{i=1}^{N} \sum_{n=-\infty}^{\infty} \imath \pi A_{n}^{i} H_{n}^{(1)}\left(k\left|\vec{r}-\vec{r}_{i}\right|\right) e^{i n \phi_{\vec{r}-\vec{r}_{i}},}
\end{aligned}
$$

$N$ being the total number of scatterers

Figures 2(b) and 2(c) show the MST predictions for the SC defined above and for the case of rigid and absorbing scatterers. The blue continuous line represents the attenuation spectrum for the $\Gamma X$ direction and red dashed line represents the attenuation spectrum for the $\Gamma M$ direction. For the case of the rigid scatterers one can see the effect of the periodicity of the structure: The blue line shows the pseudogap in the $\Gamma X$ direction between 350 and $630 \mathrm{~Hz}$. This pseudogap disappears for the $\Gamma M$ direction. These results are in completely agreement with the predictions of PWE shown in Fig. 2(a). In this structure only the scattering due to the rigidity of the scatterers is playing a role in the attenuation process producing a low and angle dependent attenuation level. In the case of the absorbent scatterers one can observe the acoustic effect of the absorbent material: the average of the IL increases in the whole range of the considered frequencies.

\section{Finite element methods: Unbounded problem}

Considering the wave propagation in free space (unbounded acoustic domain) the assumption that no waves are reflected from infinity is taken. This is known as the Sommerfeld condition. The solutions of exterior Helmholtz problems that satisfy the Sommerfeld conditions are called radiating solutions. Using FEM it is only possible to obtain some approximation of the radiating solutions in unbounded domains by applying some artificial boundaries in the numerical domain. Several techniques can be used for this purpose. ${ }^{30}$ One of them, the perfectly matched layers (PML) technique, will be briefly presented in this section.

The PML method was introduced by Berenger ${ }^{31}$ and it is an efficient alternative for emulating the Sommerfeld radiation condition in the numerical solution of wave radiation and scattering problems. The method was immediately applied to different cases based on the scalar Helmholtz equation: ${ }^{32}$ acoustics, ${ }^{33}$ elasticity, ${ }^{34}$ poroelastic media, ${ }^{35}$ shallow water waves, ${ }^{36}$ other hyperbolic problems, ${ }^{37}$ etc. Here, the interest is focused on the wave propagation time-harmonic scattering problems in linear acoustics, i.e., on the scalar Helmholtz equation.

PML consists of a coordinate transformation. ${ }^{38}$ The transformation is a scaling to complex coordinates so that the new medium becomes selectively dissipative in the direction perpendicular to the interface between the PML and the physical domain. In this work, the PML domain absorbs waves in the coordinate direction $d$ following the next coordinate transformation inside the PML:

$$
d^{\prime}=\operatorname{sgn}\left(d-d_{0}\right)\left|d-d_{0}\right|^{n} \frac{L}{\delta D^{n}}(1-\imath)
$$

where $L$ is the scaled PML width, $d_{0}$ is the coordinate of the inner PML boundary, the width of the PML is $D$, and $n$ is the scaling exponent. This coordinate transformation is provided in COMSOL 3.5 .

In practice, since the PML have to be truncated at a finite distance of the domain of interest, its external boundary produces artificial reflections. Theoretically, these reflections have minor importance due to the exponential decay of the acoustic waves inside the PML. In fact, for Helmholtztype scattering problems, it was proven that the approximate solution obtained using the PML method exponentially converges to the exact solution in the computational domain as the thickness of the layer goes to infinity. ${ }^{39}$ This result was generalized using techniques based on the pole condition. $^{40}$

Figures 2(b) and 2(c) show the FEM predictions for the considered SC made of rigid or absorbing scatterers respectively. For the FEM calculations a point source has been considered and the insertion loss has been calculated following Eq. (22). The solution domain has been discretized using $3.5 \times 10^{5}$ elements. Blue dots (red open circles) represent the attenuation spectrum for the $\Gamma X(\Gamma M)$ direction. The 
difference to the results obtained with MST at high frequencies could be due to the low number of elements in the mesh of FEM.

\section{LABORATORY EXPERIMENT}

All the experimental results shown in this work have been measured under controlled conditions in an anechoic chamber. A picture of the designed definite scatterers and a scheme of the anechoic chamber are shown in Fig. 3. In Fig. 3(b) pictures of both the periodic array of these scatterers and of the robotized acquisition hardware are also shown.

All the acoustic measurements were received in a prepolarized free-field microphone $1 / 2$ in. type $4189 \mathrm{~B} \& \mathrm{~K}$. This microphone is controlled by a 3D Robotized e-Acoustic Measurement System (3DReAMS), which is a Cartesian robot with three axes $(\mathrm{X}, \mathrm{Y}, \mathrm{Z})$ installed in the ceiling of the anechoic chamber. The robot was designed to sweep the microphone through a $3 \mathrm{D}$ grid of measuring points located at any trajectory inside the echo-free chamber. The robot includes a rotatory column installed on the ceiling of the anechoic chamber, where the periodic arrays are hung in a frame.

The National Instruments cards PCI-4474 and NI PCI7334 were used together with the Sound and Vibration Toolkit and the Order Analysis Toolkit for LABVIEW for both the data acquisition and the motion of the robot. Once the robotized system is turned off and the acoustic source and the microphone are turned on, the microphone acquires the temporal signal. From this temporal signal, one can obtain the power spectra, the frequency response or the sound-level measurement.

\section{RESULTS AND DISCUSSION}

Recently some authors have specifically constructed acoustic applications using split ring resonators (SRRs) ${ }^{15}$ motivated by the fact that these devices introduce ranges of frequencies related to the resonant frequency where waves cannot propagate through the system. ${ }^{14}$ Although authors have usually considered the SRRs as 2D Helmholtz resonators, this approximation needs some special geometrical restrictions. ${ }^{41}$ The thickness of the walls or both the length and the aperture of the neck of the resonator have to follow some approximations to be considered as a Helmholtz resonator. Otherwise, one should solve the scattering problem of the isolated resonator in order to know the resonant frequency of the SRRs, as we have done in this work. So, once the resonant frequency of the SRRs is known, they could be used to create periodic arrays with attenuation bands due to resonance in the range of frequencies below the BG of the array, i.e., in the range of low frequencies. Thus, the resonance mechanism is used in our devices to create attenuation peaks in the most difficult range of frequencies, adding to both the scattering and the absorption effects.

In this section, we analyze first the propagating properties of square periodic arrays $(a=0.33 \mathrm{~m})$ of rigid SRRs (RSRRs) in order to analyze the behavior of the resonances in a periodic system, and afterwards we will analyze the combined effect scattering-resonances-absorption with absorbing SRRs (ASRRs). The size of the finite structures considered here is $4 a \times 4 a$ and the source is placed at the origin of coordinates. The SC has been placed symmetrically to the sourcereceiver line $1.5 \mathrm{~m}$ away from the source. The IL has been calculated at a point located $3 \mathrm{~m}$ from the origin of coordinates in the direction of wave propagation. The numerical predictions have been tested using experimental measurements. The RSRRs for the experimental setup have been constructed from split ring tubes of PVC (rigid) cylinders.

\section{A. Rigid split ring resonators (RSRR)}

The RSRRs analyzed in this section have been designed with the following parameters: external radius $r=0.1 \mathrm{~m}$, inner radius $r=0.095 \mathrm{~m}$, and aperture width $L=0.02 \mathrm{~m}$. First, we have analyzed the scattering problem of an isolated RSRR in order to observe the behavior of the resonant frequency.

A wave impinging the RSRR from the left is considered, presenting the RSRR its aperture in this side, as one can see in the inset of Fig. 4. The IL produced by an isolated RSRR has been numerically obtained using FEM. Figure 4(a) shows a clear resonance peak around $220 \mathrm{~Hz}$. The open blue circles represent the experimental measurements of the IL in good agreement with the numerical predictions. Also, the localization of the pressure inside the cavity for this resonant frequency can be observed in the inset of Fig. 4(b). We note that, if the usual formula of the $2 \mathrm{D}$ Helmholtz resonator ${ }^{42}$ is used for the RSRRs presented here, the first resonant mode should appear at a frequency of $610 \mathrm{~Hz}$, which is far away from the obtained in our analysis. This fact shows that the
(A)

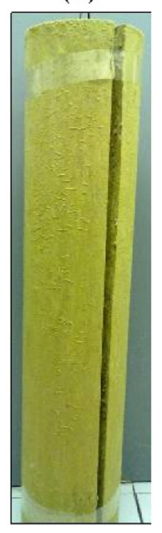

(B)

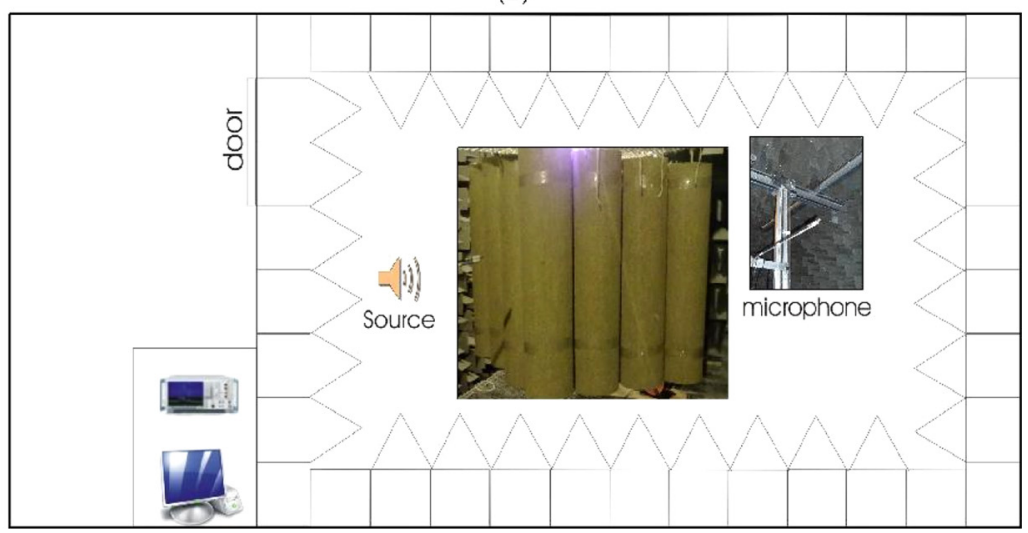

FIG. 3. (Color online) Experimental setup. (a) Picture of the single designed scatterer. (b) Scheme of the anechoic chamber, and pictures of both the experimental device designed and the robotized system 3DReAMS. 
(A)

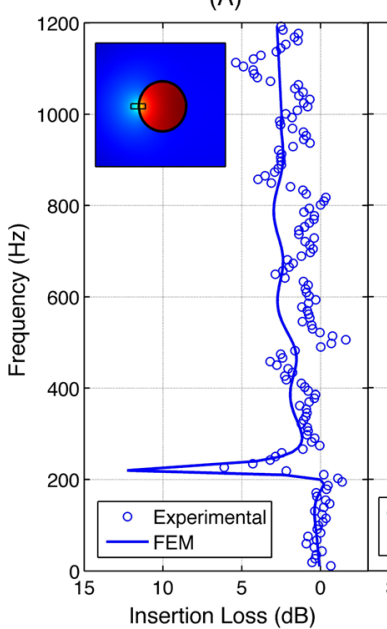

(B)

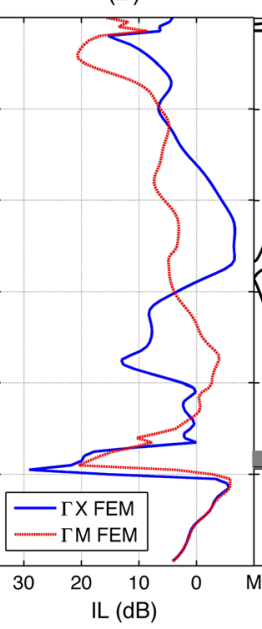

(C)

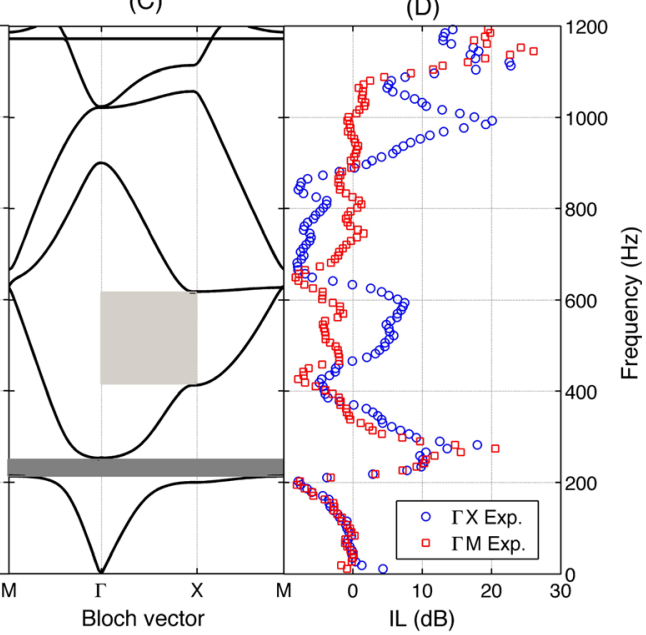

FIG. 4. (Color online) Acoustic properties of both a single and a periodic array of RSRR. (a) Numerical (blue continuous line) and experimental (blue open circles) IL produced by an isolated RSRR. (c) Dispersion relation of a square periodic array with $a=0.33 \mathrm{~m}$ made of RSRR. Strong gray area represents the attenuation band due to the resonance and weak gray area represents the pseudogap at $\Gamma X$ direction. (b) and (d) are the numerical and experimental IL of a SC made of RSRR, respectively. Blue continuous and red dashed lines in (b) [open blue circles and open red squares in (d)] are the IL in the $\Gamma X$ and $\Gamma M$ directions, respectively.

considered RSRR does not behave as a 2D Helmholtz resonator.

The band structure of the square array formed by the designed RSRR has also been numerically calculated and represented in Fig. 4(c). This diagram shows the existence of an attenuation band in the range of low frequencies (around $220 \mathrm{~Hz}$ ) due to the resonance effect of the RSRRs, independent of the BG of the array (centered at $515 \mathrm{~Hz}$ ). The numerical IL results are presented in Fig. 4(b) with the blue line (red dashed line) representing the IL at $\Gamma X(\Gamma M)$ direction. The scattering problem reproduces both the first pseudogap in the $\Gamma X$ direction and the resonance of the RSRR. On the other hand, note that these results predict the nonexistence of a pseudogap in the $\Gamma M$ direction. This fact shows the dependence of the array effects on the incidence direction. However, one also can see that the resonance effect is independent of the incidence direction: both red $\left(45^{\circ}\right)$ and blue $\left(0^{\circ}\right)$ lines show the same peak at low frequencies.

Figure 4(d) shows in blue open circles (red open squares) the experimental measurements of the IL for the considered device in the $\Gamma X(\Gamma M)$ direction. The good agreement with the theoretical predictions allow us to validate the results and demonstrates the appropriate choice of the theoretical tools to analyze this problem.

\section{B. Absorbent split ring resonators (ASRR)}

The last step of our study is the analysis of the periodic distribution of ASRRs shown in Fig. 1. In this case the three attenuation phenomena named at the beginning are considered. So, the scatterers are RSRR covered with a layer of absorbent material with a thickness $t=0.04 \mathrm{~m}$, and the designed device consist of a set of ASRRs arranged in the square array defined previously.

The study of the propagating properties of this SC is shown in Fig. 5. First of all, we have analyzed both the array and the resonant effects using the PWE method. Figure 5(b) shows the bands structure of the previous SC made of RSRRs with $r=0.1 \mathrm{~m}$ (blue dotted lines). Although the bands structure is only valid for the case of rigid cylinders (RSRRs), we have calculated the dispersion relation for the ASRR case (red continuous line) supposing this device formed by rigid scatterers $\left(r_{\text {ext }}=0.14 \mathrm{~m}\right.$, being the inner radius $r_{\text {int }}=0.095 \mathrm{~m}$ ). This strategy allows us to predict the variation of the attenuation produces in the array due to an increasing of the radius of the scatterers. If we compare both cases, the bands structure for the ASRR device predicts a complete BG centered in $515 \mathrm{~Hz}$, due to the increasing of the $f f$, that does not appear in the case of the RSRR SC. So, in
(A)

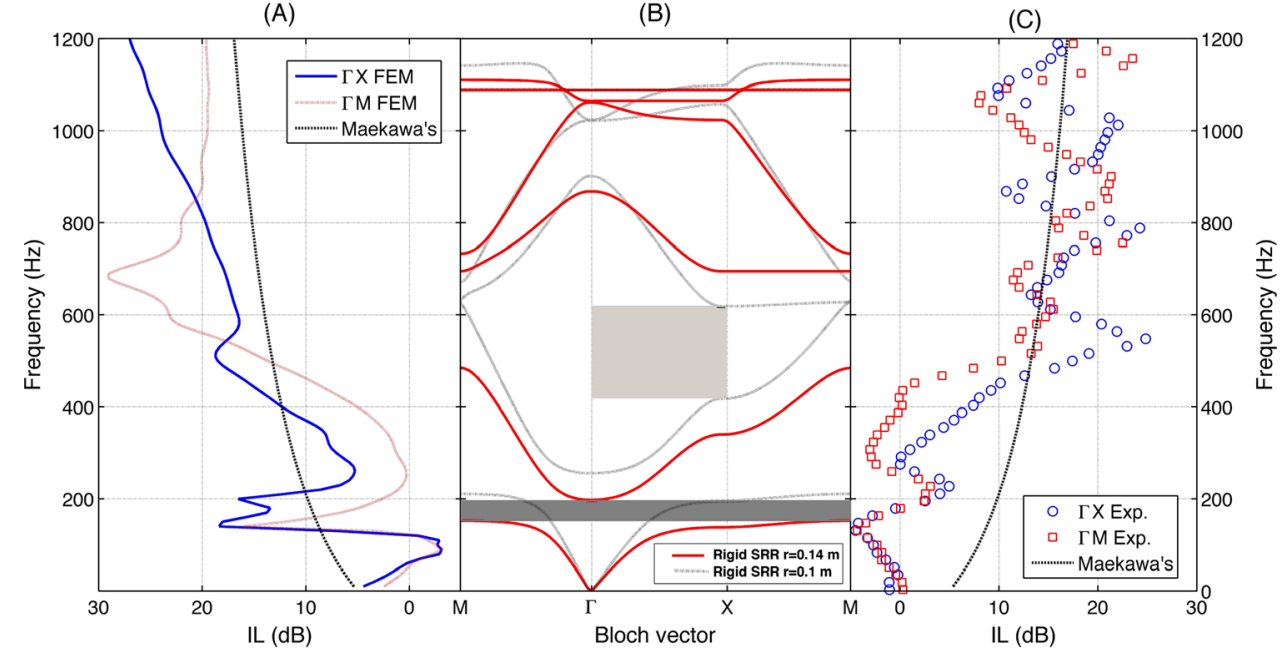

FIG. 5. (Color online) Study of the acoustical properties of a ASRR SC. (b) Dispersion relation of an square periodic array with $a=0.33 \mathrm{~m}$ made of SRR with both $r=0.1 \mathrm{~m}$ (black dashed lines) and $r=0.14 \mathrm{~m}$ (red continuous line). Strong gray area represents the attenuation band due to the resonance, and weak gray area represents the pseudogap at $\Gamma X$ direction. (a) and (c) represent the numerical and experimental IL of a SC made of ASRR, respectively. Blue continuous and red dashed lines in (a) [open blue circles and open red squares in (c)] represent the IL at $\Gamma X$ and $\Gamma M$ directions, respectively. In all cases, the dotdashed black line represents the attenuation level predicted using Maekawa's model for a classical barrier. 
the ASRR case there is no propagation at any direction for this range of frequencies.

On the other hand, as we have shown before, the resonant effect in the RSRR SC introduces an attenuation band in the low frequency range below the $\mathrm{BG}[\nu=220 \mathrm{~Hz}$, see Fig. 4(c)]. However, the corresponding attenuation band for the ASRR SC case (red continuous line) shown in Fig. 5(b) is shifted in frequency to the lower frequencies. This interesting effect is produced because the absorbent covering becomes part of the resonator, increasing the wall thickness and producing a shifting of the resonance frequency. This result can be used as a design tool that could be exploited to attenuate other near ranges of frequencies.

To determine the effect of the absorbent covering we have calculated numerically the IL of the ASRR SC. Comparing the IL levels in Figs. 5(a) and 4(b), one can observe that the average IL produced here is increased by the absorbing covering practically in the whole range of frequencies and for both $\Gamma X$ and $\Gamma M$ directions. Thus, the absorbing material introduces a baseline of attenuation independent of the angle of incidence of the wave. Although the scattering problem has been solved for the ASRR case and the bands structure is only valid for the case of rigid scatterers, one can compare both results to observe whether or not the absorbent covering destroys both the scattering and the resonance effects. Thus, in Fig. 5(a) one can observe two attenuation peaks due to both the multiple scattering at $\Gamma X$ and $\Gamma M$ directions and the resonance effect over the baseline of attenuation produced by the absorbing covering. Both peaks have been predicted by the bands structure. The experimental results are shown in Fig. 5(c). One can again observe the good agreement between the theoretical and experimental results.

Finally note that, as in the case of RSRR SCs, the attenuation level depends on the number of scatterers. We have observed that the greater the number of rows, the higher the IL. Obviously, this result is in agreement with the mass law. However, it does not seem obvious that both the multiple scattering and the resonance phenomena continue to present the same properties as in the RSRR case when the absorbent material is introduced. We have also observed that these both attenuation effects are also increased with the number of ASRR. Of course, the attenuation peak due to the resonant effect is independent of the incident direction as in the RSRR case.

\section{APPLICATIONS}

The IL of a SC made of ASRR is characterized mainly by three acoustical properties: (i) a high attenuation baseline; (ii) the structure preserve the properties of the periodicity, meaning that, it preserves the BG although the absorbing covering is surrounding the scatterers; (iii) the resonances of each scatterer are also preserved in the structure. Then, multiple scattering, resonances, and absorption coexist in the same structure without negative interference between them. Thus, the frequency response of the system in the audible acoustic frequencies is similar to a wideband bandstop filter that allows the transmission of some frequency ranges but prevent others, attenuated to very low levels.

Sound manipulation is perhaps the most obvious application of the structures presented in this work. Recently, increasing interest in such systems has motivated several works showing valuable technological applications for such an SC as acoustic filters. One can built natural SCs made of periodic arrangement of trees ${ }^{43}$ or one can even use the evanescent behavior of waves inside the SC to obtain an effective width to attenuate waves. ${ }^{23,44}$ On the other hand, one can apply optimization algorithms as, for example, evolutionary algorithms to look for the best distribution of scatterers to attenuate predetermined frequencies. ${ }^{10}$ Taking into account that bandstop filters are commonly used to eliminate particular frequencies of noise, we apply this system to develop a device as an alternative to the conventional acoustic barriers with several properties from the acoustical point of view but also with additional esthetic and constructive characteristics.

The IL of the designed device is comparable with that predicted by Maekawa for classical barriers, as one can see in Figs. 5(a) and 5(c): there are ranges of frequencies in which the SC produces better attenuation and ranges in which the SC works worse than the classical barriers. Thus, SCs made of ASRRs are suitable to be used as acoustic barriers in certain ranges of frequencies. The many technological possibilities because of the control of the multiple scattering, resonances, and absorption phenomena, as well as the nondependence of the IL with the incident angle gives additional different properties than conventional acoustic barriers.

In Fig. 6 we show two pictures of both a conventional acoustic barrier and a possible acoustic barrier based on SCs. Although the acoustic barrier based on SCs could be wider and more expensive (depending on the materials)

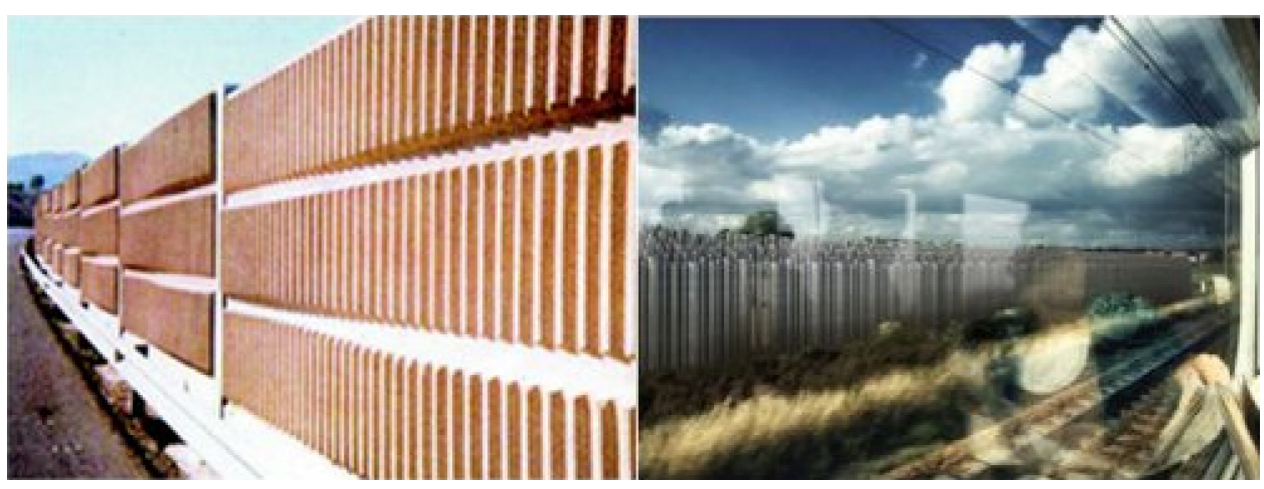

FIG. 6. (Color online) (a) Picture of a conventional acoustic barrier. (b) Simulated acoustic barrier based on SC. 
than the conventional barriers they can be attractive for some purposes because they are transparent to water and wind, presenting tunable acoustical properties, and they seem to be esthetically and constructively better than the classical ones.

\section{CONCLUSIONS}

Scatterers made of rigid walls presenting resonant cavities and covered with absorbent materials are used in this work to design a periodic distribution of ASRRs. This SC shows a constructive superposition of three physical phenomena and its attenuation properties are tunable in a wideband of frequencies by changing the parameters of the array, the characteristics of the resonant cavity, or the thickness and acoustical properties of the absorbent material. Multiple scattering theory, plane wave expansion, and finite element methods are used to theoretically study the physical behavior of the structure. Experimental results obtained in an anechoic chamber are in good agreement with both the analytical and numerical predictions. The many possibilities to control the audible noise by means of scattering, resonances, and absorption give the possibility to design a wideband bandstop acoustic filter specifically indicated to attenuate audible noise. The designed structures produces attenuation levels large enough to compete with the conventional acoustic barriers introducing high technological procedures in their design and opening the possibility to fabricate custom-tailored bandstop acoustic filters based on ASRR SCs.

\section{ACKNOWLEDGMENTS}

This work was supported by MEC (Spanish Government) and FEDER funds, under Grant No. MAT2009-09438.

${ }^{1}$ E. Yablonovitch, Phys. Rev. Lett. 58, 2059 (1987).

${ }^{2}$ S. John, Phys. Rev. Lett. 58(23), 2486 (1987).

${ }^{3}$ M. M. Sigalas and E. N. Economou, J. Sound Vib. 158, 377 (1992).

${ }^{4}$ M. S. Kushwaha, P. Halevi, L. Dobrzynski, and B. Djafari-Rouhani, Phys. Rev. Lett. 71(13), 2022 (1993).

${ }^{5}$ M. M. Sigalas, E. N. Economou, and M. Kafesaki, Phys. Rev. B 50, 3393 (1994).

${ }^{6}$ E. N. Economou and M. M. Sigalas, Phys. Rev. B 48(18), 13434 (1993).

${ }^{7}$ R. Martínez-Sala, J. Sancho, J. V. Sánchez, V. Gómez, J. Llinares, and F. Meseguer, Nature (London) 378, 241 (1995).

${ }^{8}$ J. V. Sánchez-Pérez, C. Rubio, R. Martínez-Sala, R. Sánchez-Grandia, and V. Gómez, Appl. Phys. Lett. 81, 5240 (2002).
${ }^{9}$ Yun Lai, Xiangdong Zhang, and Zhao-Qing Zhang, J. Appl. Phys. 91(9), 6191 (2002).

${ }^{10}$ V. Romero-García, J. V. Sánchez-Pérez, L. M. Garcia-Raffi, J. M. Herrero, S. García-Nieto, and X. Blasco, J. Acoust. Soc. Am. 125(6), 3774 (2009).

${ }^{11}$ J. M. Herrero, S. García-Nieto, X. Blasco, V. Romero-García, J. V. SánchezPérez, and L. M. Garcia-Raffi, Struct. Multidisc Optim. 39, 203 (2009).

${ }^{12}$ S. Castiñeira-Ibáñez, V. Romero-García, J. V. Sánchez-Pérez, and L. M. Garcia-Raffi, EPL 92, 24007 (2010).

${ }^{13}$ O. Umnova, K. Attenborough, and C. M. Linton, J. Acoust. Soc. Am. 119(1), 278-284 (2006).

${ }^{14}$ A. B. Movchan and S. Guenneau, Phys. Rev. B 70, 125116 (2004).

${ }^{15}$ Xinhua Hu and C. T. Chan, Phys. Rev. E 71, 055601 (2005).

${ }^{16}$ J. Sánchez-Dehesa, Victor M. Garcia-Chocano, D. Torrent, F. Cervera, and S. Cabrera, J. Acoust. Soc. Am., 129, 1173 (2011).

${ }^{17}$ A. Krynkin, O. Umnova, A.Y.B. Chong, S. Taherzadeh, and K. Attenborough, J. Acoust. Soc. Am. 128, 3496 (2010).

${ }^{18}$ J. B. Pendry, A. J. Holden, W. J. Stewart, and I. Youngs, IEEE Trans. Microw. Theory Technol. 47, 2975 (1999).

${ }^{19}$ E. Yablonovitch and T. J. Gmitter, Phys Rev Lett 63(18), 1950 (1989).

${ }^{20}$ D. M. Meade, K. D Brommer, A. M. Rappe, and J. D. Joannopoulos, Appl Phys Lett 61(4), 495 (1992).

${ }^{21}$ M. S. Kushwaha, P. Halevi, G. Martnez, L. Dobrzynski, and B. DjafariRouhani, Phys. Rev. B 49(4), 2313 (1994).

${ }^{22}$ V. Laude, Y. Achaoui, S. Benchabane, and A. Khelif, Phys. Rev. B 80, 092301 (2009).

${ }^{23}$ V. Romero-García, J. V. Sánchez-Pérez, and L. M. Garcia-Raffi, J. Appl. Phys. 108, 044907 (2010).

${ }^{24}$ M. I. Hussein, Phys. Rev. B 80, 212301 (2009).

${ }^{25}$ M. I. Hussein and M. J. Frazier, J. Appl. Phys. 108, 093506 (2010).

${ }^{26}$ R. P. Moiseyenko and V. Laude, Phys. Rev. B 83, 064301 (2011).

${ }^{27}$ V. Romero-García, J. V. Sánchez-Pérez, and L. M. Garcia-Raffi, New J. Phys. 12, 083024 (2010).

${ }^{28}$ V. Tournat, V. Pagneux, D. Lafarge, and L. Jaouen, Phys Rev E 70, 026609 (2004).

${ }^{29}$ You Yu Chen and Zhen Ye, Phys. Rev. E 64, 036616 (2001).

${ }^{30} \mathrm{~F}$. Ihlenburg, Finite Element Analysis of Acoustic Scattering (Springer Verlag, New York, 1998).

${ }^{31}$ J. P. Berenguer, J. Comput. Phys. 114, 185 (1994).

${ }^{32}$ I. Harari, M. Slavutin, and E. Turkel, J Comput. Acoust. 8, 121 (2000).

${ }^{33}$ Q. Qi and T. L. Geers, J. Comput. Phys. 139, 166 (1998).

${ }^{34}$ U. Basu and A. K. Chopra, Computer Methods in Applied Mechanics and Engineering 192, 1337-1375 (2003).

${ }^{35}$ Y. Q. Zeng, J. Q. He, and Q. H. Liu, Geophysics 66, 1258 (2001).

${ }^{36}$ I. M. Navon, B. Neta, and M. Y. Hussaini, Monthly Weather Rev. 132, 1369 (2004)

${ }^{37}$ J. L. Lionsand J. Mtral, and O. Vacus, Numer. Math. 92, 535 (2002).

${ }^{38}$ Q. H. Liu, J. Acoust. Soc. Am. 105, 2075 (1999).

${ }^{39}$ M. Lassas and E. Somersalo, Computing 60, 228 (1998).

${ }^{40}$ T. Hohage, F. Schmidt, and L. Zschiedrich, SIAM J. Math. Anal. 35, 547 (2003).

${ }^{41}$ F. P. Mechel, Formulas of Acoustics (Springer Verlag, New York, 2008).

${ }^{42} \nu_{\text {Helmholtz }}=(c / 2 \pi) \sqrt{A / L S}$, where $\mathrm{A}$ is the aperture, $\mathrm{L}$ is the wall thickness, and $S$ is the surface of the cavity

${ }^{43}$ R. Martínez-Sala, C. Rubio, L. M. Garcia-Raffi, J. V. Sánchez-Pérez, E. A. Sánchez-Pérez, and J. Llinares, J. Sound Vib. 291, 100 (2006).

${ }^{44}$ V. Romero-García, J. V. Sánchez-Pérez, S. Castiñeira-Ibáñez, and L.M. Garcia-Raffi, Appl. Phys. Lett. 96, 124102 (2010). 